\title{
VEHICLE PLATOON MEMBERSHIP DEFINITION FOR UNSATURATED CONDITIONS
}

\author{
Jelena Kajalić ${ }^{1}$, Nikola Čelar ${ }^{2}$, Stamenka Stanković ${ }^{3}$, Anica Kocić ${ }^{4}$ \\ 1,2,3,4 University of Belgrade, Faculty of Transport and Traffic Engineering, Vojvode Stepe 305, \\ 11000 Belgrade, Serbia
}

Received 27 December 2020; accepted 21 January 2021

\begin{abstract}
At signalized intersections, during the queue discharge, vehicles start moving along the corridor as a platoon. In unsaturated traffic conditions, vehicles can cross the stop line even after the queue discharge. In some cases, these vehicles can be members of the platoon. This paper proposes the application of logistic regression in the vehicle membership definition for unsaturated traffic conditions. The proposed model is based and tested on real traffic data.
\end{abstract}

Keywords: platoon vehicle, unsaturated traffic flow, logistic regression.

\section{Introduction}

Traffic management, as one of the basic disciplines of traffic engineering, aims to adjust the available traffic network capacity to the representative traffic demand both spatially and temporally. Traffic management of an urban network is predominantly based on the application of traffic signals. The operation of traffic signals is based on grouping traffic demands at the approach to an intersection and their efficient servicing during the time they are allowed to pass through the intersection. The efficiency of the traffic process at the intersection is the result of the selection of the appropriate management method, the applied optimization procedures and models for calculating the parameters of the traffic signal operation.

The spatial scope of a uniformly managed network part determines the division of the system into the management of individual intersections, urban arterial roads and zones. Each of the mentioned subsystems has distinct functional characteristics, specific designing issues and an appropriate role within a single traffic management system. An individual and isolated signalized intersection represents an intersection without the impact of traffic lights from adjacent intersections, so the arrival of vehicles at the intersection can be considered to be a random event. In the urban network, intersections are at relatively short distances. Consequently, the vehicle arrival at the individual intersection is conditioned by the method and operational parameters of the signals at adjacent intersections.

In cases when the vehicle arrival at the individual intersection is conditioned by the method and operational parameters of the signals at adjacent intersections, positive management effects can be realized by coordinating the signal operation at all intersections along the arterial road. The

${ }^{1}$ Corresponding author: j.kajalic@sf.bg.ac.rs 
coordinated or harmonized signal operation can be reached by establishing fixed time relations between the displays of signals along the series of adjacent intersections. This management method increases the intersection capacity by increasing the effective green time as a consequence of eliminating the start-up lost time. Along the corridors with the coordinated signal operation, harmonization of the traffic flow speeds is obtained with the minimum number of stops. This directly decreases fuel consumption, noise level, exhaust gas emission, and improves traffic safety. In addition, the decreased number of stops has a direct impact on the decrease of travel time, as well as on the increase in the average operational speed which represents the primary indicator of the level of service on urban arterial roads.

Traffic management of urban arterial roads includes the coordination of the signal operation at the pre-defined series of intersections. At the intersections which introduce vehicles into the single management system, the vehicle arrival is random or conditioned by the signal operation at adjacent intersections which are not part of the system. A queue is formed at the approach to the entry intersection during the red signal. When the green signal appears, the queue discharge starts and vehicles enter into the single management system on the arterial road by forming a vehicle platoon.

The aim of the management process is for the formed platoon to pass through the defined managed space without stopping. In other words, the platoon leader which starts moving at the initial intersection should arrive at all following intersections for the duration of the green signal, i.e. pass the complete arterial road without stopping. In order to realize this aim, the first step should include the definition of the vehicle platoon. Defining the platoon means determining the criteria for the vehicle platoon membership, based on which the platoon size is further determined.

The largest number of studies defines a platoon as a group of vehicles travelling at headways less than the defined critical headway (Athol, 1968; Mashros et al., 2014; Praveen \& Ashalatha, 2016; Paul et al., 2016; Missouri State, 1969; El-Reedy and Ashworth, 1978). Therefore, the platoon size (the number of vehicles in the platoon) will directly depend on the defined value of the critical headway. The application of a stricter criterion, in terms of a lower critical headway value, will result in the decrease of the platoon size and vice versa. The authors who use the critical headway for defining the platoon apply various threshold values. In his research, Athol (Athol, 1968) examined different headway values expressed in seconds $(1.2 ; 1.5 ; 2.1$ and 2.7$)$ and adopted the critical value of $2.1 \mathrm{~s}$ for the volume value of $1500 \mathrm{veh} / \mathrm{h}$ per lane. Jiang et al. (2002), as well as Mashros et al. (2014), defined the critical headway in the range of the $1.5 \mathrm{~s}-3.5 \mathrm{~s}$ headway values, using $0.5 \mathrm{~s}$ steps, and adopted the unique value of 2.5 s, regardless of the volume value. Similarly, Praveen \& Ashalatha (2016) determined the critical headway value in the range from 0.4 $\mathrm{s}$ to $4 \mathrm{~s}$, with the $0.4 \mathrm{~s}$ steps, while defining the critical headway value separately for each reference point. A group of authors, who defined the platoon using the critical headway at a point or a stop line at the initial intersection, adopted the critical value of $3 \mathrm{~s}$ (Missouri State, 1969), or $4 \mathrm{~s}$ (El-Reedy and Ashworth, 1978). According to the authors, the platoon involves all vehicles who cross 
the stop line with the headway less than the mentioned one.

Different headway values applied in research indicate that there is no clear procedure for its definition (Gaur \& Mirchandani, 2001). Jiang et al. (2002) stated that the critical headway should be determined on the basis of real data. The defined value of the critical headway has to provide a sufficient number of reliable data. The authors explained this position by the fact that the adoption of a lower value of the critical headway decreased the platoon size until the extreme value when the platoon could not be registered, thus decreasing the number of data required for analyzing its movement. On the other hand, the adoption of higher values of the critical headway increases the platoon size until the extreme end so that all vehicles belong to the platoon. This increases the standard deviation of certain parameters, and decreases the reliability of the obtained data. In the research (Jiang et al., 2002), (Praveen \& Ashalatha, 2016) definition of the critical headway means examining the change in the platoon size (expressed by the share in the hourly volume) and the coefficient of the platoon size for different critical headway values. The mentioned authors determined that the increase in the critical headway led to the increase in the values of both parameters until the point when they became equal. After this point, the values of both parameters rise more slowly. The headway values for which the parameters are equal is selected as the critical value.

Although the application of critical headway is the predominantly used criterion for defining the vehicle platoon, the vehicle platoon is also defined using the average headway in the literature (Chaudhary et al., 2006), (Tiaprasert, 2016). Here, defining the platoon includes observing the impact of a single headway on the average headway in the platoon. Regardless of the size of the registered platoon, the vehicle is considered a platoon member if the average headway is lower than the critical headway. Although this approach seems like a good solution, some issues might arise in the following circumstances:

- The impact of the realization of a greater headway on the average headway is directly dependent on the position of the specific vehicle. Its impact decreases with the increase of the vehicle order position, i.e. the rise of the vehicle number increases the tolerance towards the realization of greater headways.

- Realization of the same value of an individual headway in different platoons can have different results depending on the average headway value realized by previous vehicles. If the vehicles in the platoon move aggressively and realize lower average headway values, they allow for the realization of greater headway values of individual vehicles without disturbing the threshold value. If these vehicles in the platoon realize the average threshold value, not even the minimal exceeding of the threshold value will be tolerated.

\section{Description of the Problem}

When determining the platoon size, it is necessary to know the traffic process condition at the approach to the intersection. The initial queue is formed at the approach to the first intersection and it starts moving along the arterial road when the green signal appears. During the process of the queue discharge, the initial queue can be extended by the vehicles appearing at the intersection during the green signal time and joining the 
initial queue thus forming the initial platoon. In addition, after the queue discharge during the remaining green time, the stop line is crossed by the vehicles which can join the initial platoon at some situations. Therefore, it is necessary to define the conditions under which an observed vehicle can be considered to be a member of the platoon (Fig. 1).

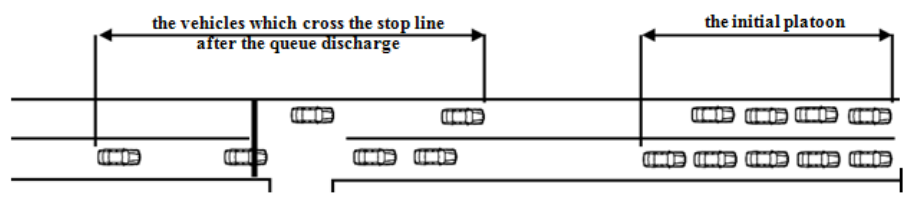

Fig. 1.

The Queue Discharge Process in Unsaturated Conditions

The following situations can occur during the queue discharge (Čelar et al., 2018):

- the queue is dissolved before the green time ends $(\mathrm{X}<1)$;

- the queue is dissolved at the end of the green time ( $\mathrm{X}=1$, threshold case);

- the queue is not dissolved during the green signal $(X>1)$.

The value $X$ (degree of saturation) represents the indicator of the traffic process condition at the signalized intersection, its approach, or the traffic lane. It is defined by the ratio of the intensity of the arrival rate and capacity.

In saturated or oversaturated conditions $(X=1 ; X>1)$, the only vehicles serviced during the green time are the vehicles from the queue formed during the red time, i.e. during the process of the queue discharge. In these cases, the vehicles from the queue leave the queue at the constant intensity of the departure rate $s$, i.e. at the saturation flow rate. Leaving the intersection at the saturation flow rate results in low headway values, which lead to the assumption that all vehicles will form the platoon. Therefore, it can be concluded that the number of vehicles in the platoon depends exclusively on the duration of the effective green time and saturation flow rate.

In unsaturated conditions $(X<1)$, when the green signal appears, the initial vehicle queue leaves the intersection at the saturation flow rate. Parallel to the process of the initial queue discharge, there is a process of vehicles joining the end of the queue. Such queue is discharged at the rate equal to the difference of the intensity of the departure and arrival rate $(s-q)$. In the unsaturated cycle, the queue discharge rate $s-q$ and the pertaining green time are such that the queue is dissolved before the green time ends. The vehicles which appear during the remaining part of the green time leave the intersection at the intensity of the arrival rate $q$ (Čelar et al., 2018). In these cases, greater headways occur most frequently after the queue discharge, which requires determining the situations in which these vehicles can be considered members of the platoon.

On the basis of the above mentioned, the vehicle platoon membership in unsaturated conditions can be defined in the following manners:

- The platoon includes only the vehicles in the queue. The platoon defined in this 
manner is problematic in the situations of continuous realization of smaller headways of the vehicles outside the queue. In these cases, these vehicles have to be considered members of the platoon.

On the other hand, the platoon can also be defined as follows:

- The platoon includes all vehicles which pass through the intersection or the reference point (stop line) during the green signal.

Although this definition was applied in some studies (Missouri State, 1969), it is problematic since in unsaturated conditions the platoon has two different parts regarding density. The headways in the first part of the platoon depend exclusively on the saturated flow value $s$, while the headways in the second part depend on the intensity of arrival rate $q$. Under real conditions, the headway of the second platoon part can vary and range from the minimal to maximal threshold values. The minimal value corresponds to the headway during the queue discharge at the saturation flow rate, while the maximum value equals the remaining green time after the queue discharge.

In unsaturated conditions, after the queue discharge, greater headways are realized in most of the cases. Therefore, a stricter criterion for the vehicle platoon membership has to be defined:

- The platoon includes all vehicles in the queue, as well as the vehicles which cross the stop line after the queue discharge while the realized headways are smaller than the pre-defined critical value.

Thus, the platoon will exclude the vehicles which crossed the stop line after the queue discharge while realizing the headway greater than the critical one. In other words, the vehicle outside the queue is a platoon member as long as the criterion regarding the headway smaller than the critical one is satisfied.

\section{Research Methodology}

The research subject of this paper involves vehicles serviced after the queue discharge, while the basic aim of the paper is to build the logistic regression model for defining the membership of these vehicles to the initial platoon. Therefore, the creation of a vehicle platoon was examined on three urban arterial roads. The research was based on recording and observing the passing of vehicles through the defined points within the research space. The research space was a section defined by the starting and ending points where the recording was performed. The starting (first) point represents the stop line at the intersection where the platoon is formed, while the ending (second) point is situated at the 200-meter distance from the stop line (Fig. 2). The recording at the defined points was conducted in the following manner:

- The first point. At the first point, the process of the queue discharge was recorded, thus registering the passing of all vehicles across the stop line during the green signal time. At the same time, a suitable Android application was used to register the passing of the last vehicle from the initial queue or the last vehicle which joined the queue (if there was one), regardless of its lane. The basic period of the research was a cycle.

- The second point. At the second point of the section, the passing of all vehicles through the observed point was recorded continuously during the complete single research period. 




Fig. 2.

Research Space at One of the Locations

After the research had been conducted, the data from the recordings were read under laboratory conditions. The data were read manually by watching the recordings from each individual point and registering the passing of each vehicle through the reference point. The passing of vehicles was observed per traffic lane using the application which registered the passing of the vehicle's rear axle over the defined point in the observed traffic lane, by pressing the field on the phone screen which corresponds to the vehicle category (Fig. 3). The subject of observation included all vehicles which entered the research space by crossing the stop line at the null point during the green time.

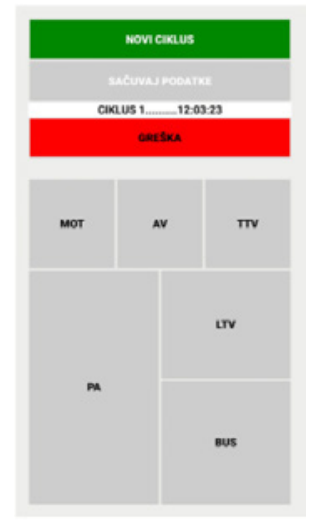

Fig. 3.

The Workspace in the Android Application

The basic set of the collected data was transferred to the standard programmes for data operation. Data reading provided the information about the cycle order, the order of the registered vehicle in the cycle, the category of the registered vehicle, the recording date and the cycle starting time, headway and cumulants.

Additional processing at the level of a point correlated data per lane at the cycle level. The data correlation was performed depending 
on the moment of the first vehicle's passing, which served as a basis for deducing the following data (Kajalić, 2020):

- The exact time of the queue discharge beginning, i.e. passing of the first vehicle through the observed point;

- The headway between the vehicles at the point per lane depending on the passing of the first vehicle (regardless of its lane);

- The total number of vehicles passing across the stop line or the observed points;

- The total number of vehicles from the initial queue, or the queue (only at the intersection);

- Headway cumulants;

- The total time of passing of all vehicles through the point within the cycle;

- Parameters of traffic signal operation (the duration of the cycle and green signal).

Bearing in mind the defined research aims, additional specific measurements were performed which helped determine whether each individual vehicle outside the queue joined the platoon at the second point.
The specific measurement procedure involved parallel watching of the recordings from the first and second point, while registering the change of each vehicle's position. The change of the position referred to the change of the traffic lane and order of the vehicle in the observed lane. Registering the position change was preceded by encoding each vehicle at the initial intersection. The encoding was performed depending on the position of the observed vehicle. For instance, the $1 \mathrm{a}$ code referred to the vehicle in the right-most lane (marked by the letter a), while number 1 indicated that the vehicle was at the first position in the observed lane. Determining the position of each individual vehicle at the following point was recorded by inserting the corresponding vehicle code into the existing database.

\section{Research Results}

After the data had been collected, the headways of all serviced vehicles were analyzed, as well as the headways of the vehicles which realized their demands after the queue discharge. The obtained results are provided in the following table (Table 1).

\section{Table 1}

Analysis of the Realized Headways of all Serviced Vehicles

\begin{tabular}{|l|l|l|l|}
\hline Analyzed Parameters & \multicolumn{1}{|c|}{ R1 } & \multicolumn{1}{|c|}{ R2 } & \multicolumn{1}{|c|}{ R3 } \\
\hline The mean value of headways of all serviced vehicles $[\mathrm{s}]$ & 2.78 & 4.28 & 3.13 \\
\hline Standard deviation of headway values of all serviced vehicles $[\mathrm{s}]$ & 2.34 & 4.39 & 3.56 \\
\hline Range of headway values of all serviced vehicles[s] & 23.01 & 31.65 & 36.16 \\
\hline Sample size - all serviced vehicles & 1203 & 540 & 1234 \\
\hline Share of the vehicles outside the queue in the total sample $(\%)$ & 32 & 31 & 10 \\
\hline
\end{tabular}

The results shown in the table (Table 1) indicate the difference in the average headway values of all serviced vehicles between the locations. The hypothesis is that the average headway depends on the percentage of vehicles which do not belong to the queue. Comparing the results obtained at the $\mathrm{R} 1$ and $\mathrm{R} 2$ locations, it can be seen that the share of vehicles which passed through the intersection after the queue discharge 
is approximately the same, while greater differences exist regarding the average headways. This leads to the conclusion that the vehicles which passed through the intersection after the queue discharge can increase the average headway, while their impact strength will depend on the type of these vehicles' arrival which can be conditioned by the traffic signal operation at the previous intersection, or random.

Table 2

Analysis of the Realized Headways of the Vehicles outside the Queue

\begin{tabular}{|l|l|l|l|}
\hline Analyzed Parameters & \multicolumn{1}{|c|}{$\mathbf{R 1}$} & \multicolumn{1}{|c|}{$\mathbf{R 2}$} & \multicolumn{1}{|c|}{$\mathbf{R 3}$} \\
\hline The mean value of headways of the vehicles outside the queue $[\mathrm{s}]$ & 1.80 & 5.26 & 2.50 \\
\hline Standard deviation of headway values of all serviced vehicles $[\mathrm{s}]$ & 1.15 & 4.07 & 3.26 \\
\hline Range of headway values of all serviced vehicles $[\mathrm{s}]$ & 12.72 & 20.25 & 21.05 \\
\hline
\end{tabular}

The comparison of the mean headway values of the vehicles outside the queue between the $\mathrm{R} 1$ and $\mathrm{R} 2$ locations confirms the results of previous studies (Table 2). On the basis of these results, it was concluded that the average headway value of the vehicles outside the queue did not depend on their share in the total demand but predominantly on the arrival manner of the vehicles from this group at the intersection.

The analysis of the research results showed that a significant percentage of the vehicles outside the queue (from $34 \%$ to $91 \%$ depending on the location) realized the headway less than $3 \mathrm{~s}$. However, on the basis of the obtained values, it cannot be stated a priori that all these vehicles will be members of the platoon, since this requires the continuous realization of the critical headway.

Realization of greater headways of the vehicles from this group does not lead directly to the conclusion that they do not belong to the platoon, since they can join the platoon in certain cases. Joining the platoon implies some of the following situations at the first point:

- The vehicle has approached the platoon;

- The vehicle has overtaken at least one vehicle from the platoon.
The vehicle is considered to have approached the platoon when its movement is conditioned by the vehicle from the platoon. According to the Highway Capacity Manual (HCM), the vehicle movement is conditioned by the movement of the preceding vehicle (the vehicle from the same traffic lane) if the realized headway value is less than or equals 3 s (HCM, 2010).

On the other hand, regardless of the realized headway, the vehicle is considered to have joined the platoon if it has overtaken at least one of the vehicles from the platoon. Apart from being dependent on the headway, the probability of joining the platoon will also depend on the position of the observed vehicle in relation to the last vehicle from the initial platoon, observed at the intersection where it has been formed.

Joining of a vehicle to the platoon was analyzed on the basis of real data obtained in the research. In the analysis the following parameters were recorded for each individual vehicle which was not a member of the queue, after the first realization of the headway greater than the defined critical value:

- Position of the observed vehicle in relation to the last vehicle in the platoon; 
- The headway of the observed vehicle in relation to the last vehicle in the platoon.

The last vehicle in the platoon is the last vehicle with the continuous realization of headways smaller than the critical one. The last vehicle in the platoon can also be a vehicle outside the queue.

On the basis of the defined criteria for joining the platoon, each observed vehicle was grouped depending on the event realization in the following way:
- Group 1 - consists of the ordered set of pairs (headway, position) for the vehicles joining the platoon;

- Group 2 - consists of the ordered set of pairs (headway, position) for the vehicles not joining the platoon.

On the basis of the obtained data, threshold values of headways for each position were determined for both groups of the vehicles. They are presented in the following graph (Fig. 4) (Kajalić, 2020).

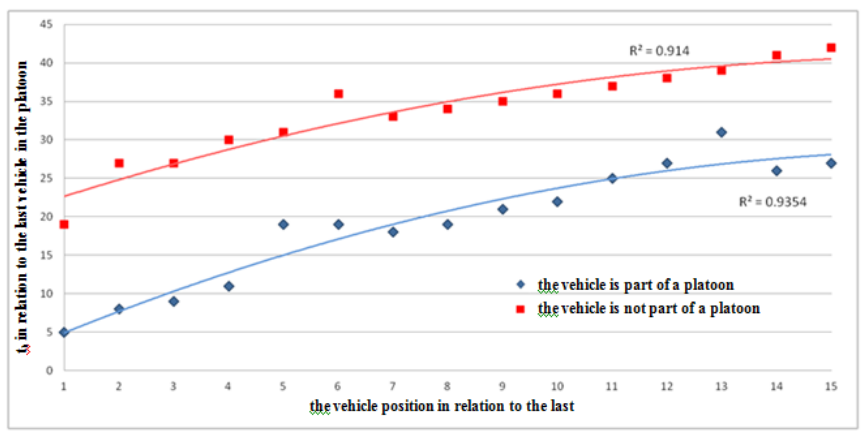

Fig. 4.

Dependence of the Vehicle Headway and Position on the Realization of Joining the Platoon

Observing all headways for the observed vehicle position which are lower than the threshold value of the first group, it can be seen that the probability of the realization of the same event (the vehicle has joined the platoon) equals 1 . On the other hand, for all headways for the observed vehicle position which are greater than the threshold value of the second group, the probability of the realization of the same event (the vehicle has not joined the platoon) equals 1 (Fig. 4). The position of vehicles and headway threshold values observed per outcome provide strong correlations described by curves (Fig. 4). The values of coefficients of determination range from 0.91 to 0.94 . On the basis of the formed curves, it can be concluded that all vehicles which realize the combination of parameters above the upper curve can be considered not to be members of the platoon, while all the vehicles with the combinations below the lower curve are considered members of the platoon. The remaining combinations of the parameters located between the two curves can yield both outcomes. 


\section{Applying the Data Classification Method for Determining the Vehicle Platoon Membership}

Deciding whether the vehicle is a member of the platoon requires the application of certain data classification methods. The classification methods are applied to make a correlation between independent and dependent variables in the situations when the output dependent variable is not a numerical value but is represented as a quantitative variable, as it is the case here (James et al., 2013). Logistic regression was applied in the paper. The method of logistic regression calculates the probability of the realization of a certain event for the combination of the known input data.

If $\mathrm{X}$ is an independent random variable on the basis of which Y should be predicted, while $Y$ can have only two values $Y=\{0,1\}$, this is called binary logistic regression.

$Y=\left\{\begin{array}{l}1, P(x) \geq q \\ 0, P(x)<q\end{array}\right.$

where $q$ represents the defined fixed value which most frequently amounts to 0.5 but can also have some other value.

In order to predict the positive outcome $(y=1)$, the logistic regression of the following form is applied (James et al., 2013):

$P(y=1 \mid x)=\frac{1}{1+e^{-\left(\beta_{0}+\beta_{1} \cdot x_{1}+\beta_{2} \cdot x_{2}+\cdots+\beta_{k} \cdot x_{k}\right)}}$

The independent variables are defined as $x$, $x_{2}, \ldots, x_{k}$, where $k$ represents the number of observed variables in the model, $\beta$ represents coefficients, with $j$ ranging from 0 to $k$.
The probability of the outcome $y=0$ is obtained as:

$$
P(y=0 \mid x)=1-P(y=1 \mid x)
$$

Further manipulating of formulas (2) and (3) provides (James et al., 2013):

$$
\begin{aligned}
& \frac{P(X)}{1-P(X)}=e^{\left(\beta_{0}+\beta_{1} \cdot x_{1}+\beta_{2} \cdot x_{2}+\cdots+\beta_{k} \cdot x_{k}\right)} \\
& \log \left(\frac{P(X)}{1-P(X)}\right)=\beta_{0}+\beta_{1} \cdot x_{1}+\beta_{2} \cdot x_{2}+\cdots+\beta_{k} \cdot x_{k}
\end{aligned}
$$

The left side of the formula (5) is called logit and depends on the linear correlation of coefficients $\beta_{j}$ and values of the independent variables. Coefficients $\beta_{j}$ are unknown and have to be estimated on the basis of the available dataset used for training the model. This represents the first phase of building the logistic regression model.

The mathematical formulation used in the procedure of determining coefficients $\beta_{j}$ is called the likelihood function and has the following form (James et al., 2013):

$l(\beta)=\sum_{i=1}^{n}\left[y_{i} \cdot \log (P(X))+\left(1-y_{i}\right) \cdot \log (1-P(X))\right]$

The presented formula is defined as the criterion function representing the sum of likelihoods for each individual input dataset. Maximizing the criterion function intends to define the values of coefficients $\beta_{j}$ so that the calculation of likelihood (2) provides the exact outcome for the largest number of input data possible. This is most frequently conducted using Newton's iterative method. Defining coefficient values enables developing of the model, which represents the second phase of building the 
logistic regression model. In the built logistic regression model, with the determined $\beta_{j}$ coefficient values, the output is the likelihood of the realization of the positive outcome which serves as a basis for further classification of the variable. The positive outcome means that $\mathrm{Y}=1$, i.e. the vehicle is a member of the platoon, while in case of $Y=0$ the vehicle is not a member of the platoon. On the basis of the built model, each new data is categorized into the more likely class.

The last, third phase involves testing the model using a known set of dependent/ independent variables, which were not used for training the model.

\section{Building the Logistic Regression Model}

As it was explained above, the procedure of building the logistic regression model contains several phases:

- $\quad$ Training the model;

- Building the model for the defined unique values of the coefficient $\beta_{j}$;

- $\quad$ Testing the model.

By means of MatLab, training the model was conducted through a series of iterations. A random dataset was selected for each iteration and used for training the model. The number of iterations depends on the sample size.

After the values of the coefficients were determined, testing of the built model was conducted using the remaining set of known values of dependent/independent variables. Also, the estimation of the model's precision was carried out, as well as the evaluation of the model's application speed reflected in the number of observations per unit of time.

In this specific case, the dataset used for building the model involved the data from three urban arterial roads (Kajalić, 2020). The data included the information related to the position of each vehicle and the headway in relation to the last vehicle in the platoon, as well as the final outcome according to which the vehicle joined or did not join the platoon. The last vehicle in the platoon represents the last vehicle in the queue. Logistic regression was applied only to the vehicles which were not part of the queue from the moment of the first realization of the headway greater than the defined critical value.

The event outcome (the vehicle joined the platoon or the vehicle did not join the platoon) was known for each observed vehicle. The realizations of possible outcomes were coded using the value 1 if the vehicle joined the platoon or 0 in the opposite case.

In order to create the dataset, it was necessary to define the values of the critical headway. The model was tested for three values of the critical headway ( $3 \mathrm{~s}, 2.5 \mathrm{~s}$ and $2.1 \mathrm{~s}$ ). The selection of the critical headway value was conducted on the basis of the literature review (Athol, 1968), (Praveen \& Ashalatha, 2016), (Mashros et al., 2014).

Table 3

The Obtained Model Precisions for Different Values of the Critical Headway $t_{h}$

\begin{tabular}{|l|c|c|c|}
\hline Critical Headway & $\mathbf{3 ~ s}$ & $\mathbf{2 . 5} \mathbf{~ s}$ & $\mathbf{2 . 1} \mathbf{~ s}$ \\
\hline $\mathbf{N}$ & 508 & 557 & 626 \\
\hline Model Precision & 0.82 & 0.82 & 0.83 \\
\hline
\end{tabular}


Table 1 shows the precision values of the applied model depending on the adopted value of the critical headway. The change of the critical headway value alters the precision of the obtained model. It should be mentioned that the change of the critical headway consequently changes the sample size $(\mathrm{N})$, i.e. the stricter the criteria the larger the sample (Table 3 ). The model's precision depends on the model's sensitivity and specificity. The model's sensitivity refers to the accuracy of classification for $y=1$, while the model's specificity is related to the accuracy of classification for $\mathrm{y}=0$.

Table 4

Quality of Separability Depending on the AUC Value

\begin{tabular}{|l|c|}
\hline AUC & \\
\hline $\mathbf{0 . 5}$ & No separability \\
\hline $\mathbf{0 . 5 - 0 . 7}$ & Poor separability \\
\hline $\mathbf{0 . 7 - 0 . 8}$ & Acceptable separability \\
\hline $\mathbf{0 . 8}-0.9$ & Excellent separability \\
\hline$>\mathbf{0 . 9}$ & Outstanding separability \\
\hline
\end{tabular}

Each point of the curve represents an ordered pair (sensitivity, specificity) corresponding to the individual decision level. The AUC estimation amounting to 0.5 corresponds to the theoretical curve for the test with no separability (identical distribution of results of the two groups), and it represents the line connecting the ordered pair $(0,0)$ and $(1,1)$. The AUC estimation amounting to 1 represents a
The comparison of the obtained results shown in the table (Table 3) indicates that the decrease of the critical headway leads to the increase in the model's precision. However, an additional analysis was conducted prior to selecting the critical headway.

The applied measure for estimating the precision of classification was C-Statistic or AUC (Area Under Curve) representing the area below the ROC (Receiver Operating Characteristic) curve. The values of the area under the curve range from 0.5 to 1 (Peng et al., 2002).

\section{Table 5}

The AUC Values of the Areas under the ROC Curve for Different Values of the Critical Headway $t_{h}$

\begin{tabular}{|l|c|c|c|}
\hline Critical Headway & $\mathbf{3 ~ s}$ & $\mathbf{2 . 5} \mathbf{~ s}$ & $\mathbf{2 . 1} \mathbf{~ s}$ \\
\hline AUC & 0.79 & 0.84 & 0.86 \\
\hline
\end{tabular}

The table shows the comparison of the obtained ROC curves for different values of the critical headway and the areas obtained under them for each of the proposed models. line parallel to the $\mathrm{x}$-axis, while it crosses the $y$-axis in 1 . In real cases, ROC curves are located between these threshold values, and in terms of quality the one closer to the upper left corner indicates the test with a higher precision.

The AUC values of the areas under the ROC curve for different critical headway values are shown in the following table (Table 5).
The results show the acceptable separability for the critical headway value of $3 \mathrm{~s}$, while excellent separability is obtained for the critical headway values of 2.5 and $2.1 \mathrm{~s}$. 
On the basis of the presented analysis of the results by applying the logistic regression for different values of the critical headway, it can be concluded that the model with the applied headway of $2.1 \mathrm{~s}$ shows the best fit to the data. Therefore, this model is adopted for determining the vehicle platoon membership.

The adopted logistic regression model for defining the vehicle platoon membership is used to calculate the realization of the positive event outcome. The adopted model has the following final form:

$$
P(x)=\frac{1}{1+e^{-\left(2.773106+0.419244 \cdot x_{1}-0.21247 \cdot x_{2}\right)}}
$$

where $P(x)$ is the probability of realization of the positive outcome of the event, $x_{1}$ is the headway of the observed vehicle expressed in seconds and $x_{2}$ is the vehicle position in relation to the last vehicle in the platoon.

\section{Conclusion}

The efficient traffic process on urban arterial roads, whose transport demand volume makes them the core of the urban traffic system operation, represents the primary aim of traffic management. The coordinated operation of traffic signals represents the basic strategy for reaching this aim. In order to achieve the positive management effects of applying the coordinated signal operation, it is requisite to know a series of parameters. In this sense, the parameter of the platoon size is of great significance.

In unsaturated conditions, there are greater headways between the vehicles which realize their demand after the queue discharge. In certain situations, these vehicles can join the platoon, and the probability of their joining will depend on the realized headway and the vehicle position in relation to the last vehicle in the platoon. The paper presents the research which has built a logistic regression model for determining the vehicle platoon membership. On the basis of the presented analysis of the results by applying the logistic regression for different critical headway values, it can be concluded that the model with the adopted critical value of 2.1 $s$ provides the best fit to the data and will have the good estimation in $83 \%$ of the cases.

For the efficient coordination of traffic signal operation, the platoon size should be known. In addition, the characteristics of the platoon movement should also be defined. Therefore, future research could be directed at determining the characteristics of the platoon movement.

\section{Acknowledgement}

This paper is a part of the project funded by the Ministry of Education, Science and Technological Development of the Republic of Serbia (project TR 36021).

\section{References}

Athol, P. 1968. Headway groupings, Highway Research Record (72): 137-155. Available from Internet: <http:// onlinepubs.trb.org/Onlinepubs/hrr/1965/72/72-007. $\mathrm{pdf}>$.

Čelar, N.; Stanković, S.; Kajalić, J. 2018. Traffic Signal Settings [In Serbian: Osnove upravljanja svetlosnim signalima]. Faculty of Transport and Traffic Engineering, University of Belgrade, Belgrade. 181p.

Chaudhary, N.; Abbas, M.; Charara, H. 2006. Development and Field Testing of Platoon Identification and Accommodation System, Transportation Research Record 1978(1): 141-148. doi: https://doi. org/10.3141/1978-19. 
El-Reedy, T.Y.; Ashworth, R. 1978. Platoon Dispersion Along a Major Road in Sheffield, Traffic Engineering and Control 19(4): 186-189.

Gaur, A.; Mirchandani, P. 2001. Method for Real-Time Recognition of Vehicle Platoons, Transportation Research Record 1748(1): 8-17. doi: https://doi.org/10.3141/174802.

HCM. 2010. Highway Capacity Manual (Vol. 4). Research Board, National Research Council, Washington, D.C.

James, G.; Witten, D.; Hastie, T.; Tibshirani, R. 2013. An Introduction to Statistical Learning. Springer, New York, USA. 426p. doi: https://doi.org/10.1007/978-1-46147138-7.

Jiang, Y.; Li, S.; Shamo, D. 2002. Development of vehicle platoon distribution models and simulation of platoon movements on Indian rural corridors. Report No. FHWA/IN/JTRP-2002/23, Federal Highway Administration U.S. Department of Transportation. 85p.

Kajalić, J. 2020. Vehicle Platoon Moddeling on Arterial Street [In Serbian: Modeliranje kretanja plotuna vozila na gradskim arterijama]. Doctoral disertation, Faculty of Transport and Traffic Engineering, University of Belgrade, Belgrade. 105p.
Mashros, N.; Hainin, M.R.; Hassan, N.A.; Yunus, N.Z.M.; Kadir, M.A.A. 2014. Exploring the pattern of platoon dispersion caused by traffic signal, Jurnal Teknologi 71(3): 7-13. doi: https://doi.org/10.11113/jt.v71.3751.

Missouri State. 1969. Platoon dispersion at selected distances from signalized intersections. Report no. 68-6 Federal Highway Administration U.S. Department of Transportation, USA.

Paul, B.; Mitra, S.; Maitra, B. 2016. Calibration of Robertson's platoon dispersion model in non-lane based mixed traffic operation, Transportation in Developing Economies 2(2): 1-14. doi: https://doi.org/10.1007/ s40890-016-0016-7.

Peng, C.Y.J.; Lee, K.L.; Ingersoll, G.M. 2002. An Introduction to Logistic Regression Analysis and Reporting, The Journal of Educational Research 96(1): 3-14.

Praveen, P. S.; Ashalatha, R. 2016. Platoon diffusion along signalized corridors, Procedia Technology 25: 154161. doi: https://doi.org/10.1016/j.protcy.2016.08.092.

Tiaprasert, K. 2016. Queue length estimation and platoon recognition using connected vehicle technology for adaptive signal control. Doctoral disertation, Texas A\&M University, USA. 106p. 